\title{
Holomorphic Extensions of Formal Objects
}

\author{
JAVIER RIBÓN
}

\begin{abstract}
We are interested on families of formal power series in $(\mathbb{C}, 0)$ parameterized by $\mathbb{C}^{n}\left(\hat{f}=\sum_{m=0}^{\infty} P_{m}\left(x_{1}, \ldots, x_{n}\right) x^{m}\right)$. If every $P_{m}$ is a polynomial whose degree is bounded by a linear function ( $\operatorname{deg} P_{m} \leq A m+B$ for some $A>0$ and $B \geq 0)$ then the family is either convergent or the series $\hat{f}\left(c_{1}, \ldots, c_{n}, x\right) \notin \mathbb{C}\{x\}$ for all $\left(c_{1}, \ldots, c_{n}\right) \in \mathbb{C}^{n}$ except a pluri-polar set. Generalizations of these results are provided for formal objects associated to germs of diffeomorphism (formal power series, formal meromorphic functions, etc.). We are interested on describing the nature of the set of parameters where $\hat{f}=\sum_{m=0}^{\infty} P_{m}\left(x_{1}, \ldots, x_{n}\right) x^{m}$ converges. We prove that in dimension $n=1$ the sets of convergence of the divergent power series are exactly the $F_{\sigma}$ polar sets.
\end{abstract}

Mathematics Subject Classification (2000): 32D15 (primary); 31A15, 32S65, 40A05 (secondary).

\section{1. - Introduction}

There are some dynamical systems with associated formal fibered diffeomorphisms; for instance formal linearizing applications related to small divisors problems. We study series of the form

$$
\hat{f}\left(x_{1}, \ldots, x_{n}, x\right)=\sum_{m=0}^{\infty} P_{m}\left(x_{1}, \ldots, x_{n}\right) x^{m} \quad \text { where } P_{m} \in \mathbb{C}\left[x_{1}, \ldots, x_{n}\right]
$$

and $\operatorname{deg} P_{m} \leq A m+B$ for some $A>0$ and $B \geq 0$. We denote the set of such series as $\mathbb{C}\left[x_{1}, \ldots, x_{n}\right][[x]]_{A, B}$. We say that $\hat{f}$ converges on the line $\left(x_{1}=c_{1}\right) \cap \ldots \cap\left(x_{n}=c_{n}\right)$ if $\hat{f}\left(c_{1}, \ldots, c_{n}, x\right) \in \mathbb{C}\{x\}$. The set of lines is parameterized by $\mathbb{C}^{n}$. It is known that

Proposition 1.1. Let $\hat{f}$ be an element of $\mathbb{C}\left[x_{1}, \ldots, x_{n}\right][[x]]_{A, B}$. Then $\hat{f}$ converges in a neighborhood of $x=0$ if and only if $\hat{f}$ converges on $\left(x_{1}=c_{1}\right) \cap \ldots \cap\left(x_{n}=\right.$ $\left.c_{n}\right)$ for every $\left(c_{1}, \ldots, c_{n}\right) \in \mathbb{C}^{n}$.

The author is financed by "Secretaría de Estado de Educación y Universidades".

Pervenuto alla Redazione il 12 dicembre 2003 e in forma definitiva il 22 luglio 2004. 
We do not need to test convergence in all the lines in order to assure convergence in a neighborhood of $x=0$.

Proposition 1.2. Let $\hat{f}$ be an element of $\mathbb{C}\left[x_{1}, \ldots, x_{n}\right][[x]]_{A, B}$. If $\hat{f}$ converges on an open set $\left(\right.$ in $\left.\mathbb{C}^{n}\right)$ of lines, then $\hat{f}$ converges.

This result can be proved using elementary techniques involving topics like Baire's theorem and Cauchy's integration formula.

We can improve a lot Proposition 1.2. Nadirashvili's lemma allows to change open by Lebesgue non-null measurable sets. But the sets of positive measure are far from being optimal, for instance in dimension 1 we have

Proposition 1.3. Let $\hat{f}$ be an element of $\mathbb{C}[t][[x]]_{A, B}$. If $\hat{f}$ converges on a connected set (with more than one element) of lines, then $\hat{f}$ converges.

An elementary proof will be provided in next section. Our main tools will be Baire's theorem, Lagrange's interpolation formula and Stirling's formula. A unified approach to the previous results is possible by using complex analysis and specially potential and pluri-potential theories.

THEOREM 1.1. [5] If $\hat{f} \in \mathbb{C}\left[x_{1}, \ldots, x_{n}\right][[x]]_{A, B}$ converges on the lines contained in a non pluri-polar set (in $\left.\mathbb{C}^{n}\right)$, then $\hat{f}$ is a convergent function in the neighborhood of $x=0$.

Open sets, non-null measurable sets and connected subsets of $\mathbb{C}$ with more than one element are not pluri-polar. Theorem 1.1 is a generalization of all the previous propositions.

We say that $\hat{f} \in \mathbb{C}\left[\left[x_{1}, \ldots, x_{n}\right]\right]$ converges on a germ of curve $\gamma(t)$ if $\hat{f} \circ \gamma(t)$ converges. Theorem 1.1 has an analogue for power series in $\mathbb{C}\left[\left[x, x_{1}, \ldots, x_{n}\right]\right]$ and lines trough the origin. The application

$$
\Delta:\left(x, x_{1}, \ldots, x_{n}\right) \rightarrow\left(x, x x_{1}, \ldots, x x_{n}\right)
$$

maps lines of the form $\left(x_{1}=c_{1}\right) \cap \ldots \cap\left(x_{n}=c_{n}\right)$ to lines through the origin. Moreover, it induces an isomorphism of rings between $\mathbb{C}\left[\left[x, x_{1}, \ldots, x_{n}\right]\right]$ and $\mathbb{C}\left[x_{1}, \ldots, x_{n}\right][[x]]_{1,0}$. Since lines through the origin are parameterized by $\mathbb{P}^{n-1}(\mathbb{C})$ next corollary makes sense

COROLlaRY 1.1. If an element of $\mathbb{C}\left[\left[x_{1}, \ldots, x_{n}\right]\right]$ converges on a non pluripolar set of lines through the origin, then it does converge.

The aim of this work is to extend the previous results. There are formal objects associated to geometrical models in a natural way. As an example consider the holomorphic germs of diffeomorphism in $\left(\mathbb{C}^{n}, 0\right)$. A germ $\varphi \in$ $\operatorname{Diff}\left(\mathbb{C}^{n}, 0\right)$ whose linear part $j^{1} \varphi$ is the identity can be written in the form

$$
\varphi=\exp (\widehat{X}), \quad \widehat{X}=\sum_{i=1}^{n} \hat{a}_{i} \frac{\partial}{\partial x_{i}}, \quad \hat{a}_{i} \in \mathbb{C}\left[\left[x_{1}, \ldots, x_{n}\right]\right] \forall i \in\{1, \ldots, n\}
$$


The exponential can be defined for any formal nilpotent vector field by the formula

$$
\exp (\widehat{X})=\left(\sum_{m=0}^{\infty} \frac{\widehat{X}^{m}\left(x_{1}\right)}{m !}, \ldots, \sum_{m=0}^{\infty} \frac{\widehat{X}^{m}\left(x_{n}\right)}{m !}\right) .
$$

This formula gives the analytic expression of the exponential for convergent vector fields. The structure of the logarithm $\widehat{X}$ of a germ of diffeomorphism $\varphi(\varphi=\exp (\widehat{X}))$ is not really known. We only know that $\widehat{X}$ is likely to be summable in suitable domains.

Any formal vector field $\widehat{X}$ supports a formal 1-dimensional foliation. This foliation is identified with the class of formal vector fields generically colinear to $\widehat{X} ; \widehat{X}$ and $\widehat{Y}$ belong to the same class if there exist $\hat{f}$ and $\hat{g}$ non-zero power series such that $\hat{f} \widehat{X}=\hat{g} \widehat{Y}$. We will say that the foliation supported by $\widehat{X}$ is convergent if there is a convergent vector field generically colinear to $\hat{X}$.

The elements of the quotient field of $\mathbb{C}\left[\left[x_{1}, \ldots, x_{n}\right]\right]$ will be called formal meromorphic functions. The formal meromorphic function $\hat{f} / \hat{g}$ is convergent if there exist convergent germs of function $f$ and $g(g \neq 0)$ such that $\hat{f} g=\hat{g} f$. The formal foliation supported by a formal vector field $\widehat{X}=\sum_{i=1}^{n} \hat{a}_{i} \frac{\partial}{\partial x_{i}}$ is convergent if and only if all the formal meromorphic functions $\hat{a}_{i} / \hat{a}_{j}(\{i, j\} \subset$ $\{1, \ldots, n\})$ are convergent.

The foliation supported by the logarithm of a germ of diffeomorphism (such that $j^{1} \varphi=I d$ ) can have formal first integrals, as formal power series or formal meromorphic functions. For instance, consider

$$
\varphi=\left(x+x^{2}+x^{3}, y+y^{2}+y^{3}\right)=\exp (\widehat{X})=\exp \left(\hat{a}(x) \frac{\partial}{\partial x}+\hat{a}(y) \frac{\partial}{\partial y}\right) .
$$

Since $z \mapsto z+z^{2}+z^{3}$ is formally but not analytically conjugated to $z \mapsto z /(1-z)$, then $\varphi$ is formally conjugated to

$$
\alpha=\left(\frac{x}{1-x}, \frac{y}{1-y}\right)=\exp \left(x^{2} \frac{\partial}{\partial x}+y^{2} \frac{\partial}{\partial y}\right)
$$

but $\hat{a}(z)$ is divergent. The foliation supported by $x^{2} \frac{\partial}{\partial x}+y^{2} \frac{\partial}{\partial y}$ has a pure meromorphic first integral $(y-x) / x y$ and $\widehat{X}$ has a first integral

$$
\widehat{H}=\frac{\hat{h}(y)-\hat{h}(x)}{\hat{h}(x) \hat{h}(y)}
$$

where $\hat{h}(z)$ is the formal divergent conjugation between $z \mapsto z+z^{2}+z^{3}$ and $z \mapsto z /(1-z)$. The first integral $\widehat{H}$ is divergent (it's the product of $1 / x y$ times a divergent power series). The foliation supported by $\widehat{X}$ is divergent as $\hat{a}(x) / \hat{a}(y)$ is. 
We will provide an example of germ of diffeomorphism whose logarithm has a formal logarithmic function as a first integral. We call formal logarithmic function an expression of the form $\hat{f}_{1}^{\lambda_{1}} \ldots \hat{f}_{p}^{\lambda_{p}}$ where $\hat{f}_{i} \in \mathbb{C}\left[\left[x_{1}, \ldots, x_{n}\right]\right]$ and $\lambda_{i} \in \mathbb{C}$ for all $1 \leq i \leq p$. By definition $\hat{f}_{1}^{\lambda_{1}} \ldots \hat{f}_{p}^{\lambda_{p}}$ converges if there exist formal units $\hat{u}_{i}$ such that $\hat{u}_{i} \hat{f}_{i} \in \mathbb{C}\left\{x_{1}, \ldots, x_{n}\right\}$ for all $1 \leq i \leq p$ and $\hat{u}_{1}^{\lambda_{1}} \ldots \hat{u}_{p}^{\lambda_{p}}$ converges. This definition generalizes the notions of convergence of formal power series and formal meromorphic functions. We prove:

Proposition 1.4. Let $\hat{f}$ be a formal logarithmic function $\hat{f}_{1}^{\lambda_{1}} \ldots \hat{f}_{p}^{\lambda_{p}}$. If $\hat{f}$ converges on a non pluri-polar set of lines, then $\hat{f}$ converges.

The lines through the origin are not so special. In dimension $n=2$ we can change the radial fibration by any germ of dicritical foliation (dicritical means that the foliation has infinitely many integral curves). The set of integral curves $I(\omega)$ of a germ of foliation $\omega$ in $\left(\mathbb{C}^{2}, 0\right)$ has the structure of a finite union of analytic manifolds. This is a consequence of Seidenberg's desingularization theorem [8]; we can apply it to desingularize $\omega$ via a finite number of blow-ups. Then, the number of dicritical (i.e., generically transversal to $\omega$ ) components of the divisor $D$ is finite. For each dicritical component $S$ of $D$ we exclude a finite set $E_{S}$; more precisely $E_{S}$ is the union of the singular set of $\omega$ in $S$ and the corners of $S$ (i.e., the intersection of $S$ with the remaining components of $D$ ). The set $S \backslash E_{S}$ is biholomorphic to $\mathbb{P}^{1}(\mathbb{C})$ minus a finite set, moreover each point in $S \backslash E_{S}$ is contained in the strict transform of a unique invariant curve of $\omega$. A curve non-parameterized by any $S \backslash E_{S}$ passes through a singular point of $\omega$ in $D$. This set is finite and since the singularities are irreducible the set of remaining curves is finite.

We can define what a polar set of integral curves is because polarity is a local property. We consider $V_{1}, \ldots, V_{p}$ the one dimensional maximal manifolds contained in $I(\omega)$. We say that a formal logarithmic function $\hat{f}$ converges on a non pluri-polar set of integral curves of $\omega$ if $\hat{f}$ converges on a non pluri-polar set of curves in some $V_{i}(i \in\{1, \ldots, p\})$. In this context we prove:

PROPOSITION 1.5. Convergence of a formal logarithmic function on a non pluripolar set of integral curves implies convergence.

Instead of enlarging the objects and fibrations whom we can apply theorems of forced convergence we can characterize the sets of lines of convergence of divergent power series. We solve completely the problem in dimension $n=1$. Theorems in [3], [6], [4], [5] shows that the set of lines of convergence of an element of $\mathbb{C}[t][[x]]_{A, B}$ is either $\mathbb{C}$ or a $F_{\sigma}$ polar set. We prove in this article the reciprocal theorem.

THEOREM 1.2. Let $F$ be a $F_{\sigma}$ polar set and let $A$ and $B$ real constants such that $A>0$ and $B \geq 0$. There exists a divergent power series $\hat{f}_{F} \in \mathbb{C}[t][[x]]_{A, B}$ such that $\hat{f}_{F}$ converges on the lines in $F$ and diverges on the others. 
We really prove the theorem for $A=1$ and $B=0$. The other cases are deduced immediately from this one. The series $\hat{f}_{F}$ is quite lacunar i.e., there are plenty of zeros in the Taylor power series development of $\hat{f}_{F}$. Our theorem is a sort of Deny's theorem; Deny's theorem says that the minus infinity levels of subharmonic functions are exactly the $G_{\delta}$ polar sets. This result proves that the choice of potential and pluri-potential theories is the good one. As an immediate corollary of the theorem we obtain

Corollary 1.2. Let $F \subset \mathbb{P}^{1}(\mathbb{C})$ be a $F_{\sigma}$ polar set. There exists a divergent power series $\hat{f}_{F} \in \mathbb{C}[[x, y]]$ such that $\hat{f}_{F}$ converges on the lines in $F$ and diverges on the others.

In the last part of the article we work with series in $\mathbb{C}[t][[x]]_{A, B}$. For $A=1$ and $B=0$ the statements can be translated to $\mathbb{C}[[x, y]]$ via the change of coordinates $t=y / x$.

We want to know how a subset of $\mathbb{C}$ is approximated by $F_{\sigma}$ sets. We divide the subsets of $\mathbb{C}$ in three groups: big, small and mixed sets. A set $E$ is small if there exists a divergent power series in $\mathbb{C}[t][[x]]_{A, B}$ converging on $E$, i.e., $E$ is small if it is contained in a $F_{\sigma}$ polar set. A set $E$ is big if all the $F_{\sigma}$ sets containing $E$ have logarithmic capacities bounded below by a positive constant. The remaining sets are the mixed ones.

We define for any $\hat{f} \in \mathbb{C}[t][[x]]_{A, B}$ the complex function $r[\hat{f}](t)$ which associates to $t \in \mathbb{C}$ the radius of convergence of $\hat{f}(t, x)$.

If a series $\hat{f} \in \mathbb{C}[t][[x]]_{A, B}$ converges on $E \subset \mathbb{C}$ then we define

$$
G_{E}(\hat{f})=\inf _{t \in E}\left\{r[\hat{f}](t)\left(1+|t|^{A}\right)\right\}, \quad\left(G_{E}(\hat{f}) \in[0, \infty]\right) .
$$

The factor $1+|t|^{A}$ enters in the formula because the radius of convergence $r(t)$ of a convergent element of $\mathbb{C}[t][[x]]_{A, B}$ in a neighborhood of $x=0$ is such that [3]

$$
r(t) \geq \frac{C}{1+|t|^{A}} \text { for some constant } C>0 .
$$

We deduce that $\hat{f} \in \mathbb{C}[t][[x]]_{A, B}$ converges in a neighborhood of $x=0$ if and only if $G_{\mathbb{C}}(\hat{f})$ is bigger than 0 . We note that if $G_{\mathbb{C}}(\hat{f})>0$ then $\hat{f}$ is the power series development of an analytic function defined in $|x|<G_{\mathbb{C}}(\hat{f}) /\left(1+|t|^{A}\right)$. Theorem 1.1 proves the convergence of $\hat{f}$ in a neighborhood of $x=0$ and Hartogs lemma allows to extend $\hat{f}$ to $|x|<G_{\mathbb{C}}(\hat{f}) /\left(1+|t|^{A}\right)$. We have

Proposition 1.6. Let $E \subset \mathbb{C}$ be a big set. Then there exists a constant $C_{E}>0$ such that $G_{\mathbb{C}}(\hat{f}) \geq C_{E} G_{E}(\hat{f})$ for any power series $\hat{f} \in \mathbb{C}[t][[x]]_{A, B}$ converging on $E$.

Proposition 1.7. Let $E \subset \mathbb{C}$ be a mixed set. Then $\inf \left\{G_{\mathbb{C}}(\hat{f}) / G_{E}(\hat{f}) / \forall \hat{f} \in \mathbb{C}[t][[x]]_{A, B}\right.$ converging on $\left.E\right\}=0$.

ACKNowledgements. I am grateful to Ricardo Pérez Marco for introducing me to this subject. I thank the referee for the helpful suggestions. 


\section{2. - Motivation}

In this section we present how potential theory is related to problems of convergence. The next results contain the germ of the ideas we will use later.

Proposition 2.1. Let $\hat{f}$ be an element of $\mathbb{C}[t][[x]]_{A, B}$. If $\hat{f}$ converges on a connected set (with more than one element) of lines $E \subset \mathbb{C}$ then $\hat{f}$ is convergent in a neighborhood of $x=0$.

PROOF. We denote by $[\lambda]$ the integer part of a real number $\lambda$. Let $\hat{f}$ be $\sum_{m=0}^{\infty} P_{m}(t) x^{m}$. We can suppose, by doing a linear transformation in $t$, that 0 and 1 belong to $E$. For any $\epsilon \in(0,1)$ the intersection $E \cap(|t|=\epsilon)$ is not empty. If not, the sets $E \cap(|t|<\epsilon)$ and $E \cap(|t|>\epsilon)$ would be disjoint open non-empty subsets of $E$. That's not possible because $E$ is connected. We define for $N \in \mathbb{N}$ the sets

$$
A_{N}=\left\{t \in \mathbb{C} \text { such that }\left|P_{m}(t)\right| \leq N^{m} \quad \forall m>0\right\} .
$$

All the sets $A_{N}(N \in \mathbb{N})$ are closed. We define the set $B_{N} \subset[0,1](N \in \mathbb{N})$ as the intersection of $[0,1]$ and the image of $A_{N}$ by the application $t \mapsto|t|$. The sets $B_{N}(N \in \mathbb{N})$ are also closed. Since $\cup_{N \in \mathbb{N}} B_{N}=[0,1]$ then Baire's theorem implies that there exists $M \in \mathbb{N}$ such that the interior of $B_{M}$ is not empty. We choose an interval $\left[t_{0}, t_{0}+\epsilon\right]$ contained in $B_{M}(\epsilon>0)$. The behavior of $\hat{f}$ in $A_{M}$ will lead us to obtain global properties of the family of polynomials $P_{m}$ $(m>0)$. Let us define $h(m)=[A m+B]$; we choose $h(m)+1$ points in $A_{M}$ such that

$$
\left|a_{i}\right|=t_{0}+\frac{\epsilon}{h(m)} i \text { for all } i \in\{0, \ldots, h(m)\}
$$

Since deg $P_{m} \leq h(m)$ we use Lagrange's interpolation formula to obtain

$$
P_{m}(t)=\sum_{i=0}^{h(m)} P_{m}\left(a_{i}\right) \frac{\left(t-a_{0}\right) \ldots\left(t-a_{i-1}\right)\left(t-a_{i+1}\right) \ldots\left(t-a_{h(m)}\right)}{\left(a_{i}-a_{0}\right) \ldots\left(a_{i}-a_{i-1}\right)\left(a_{i}-a_{i+1}\right) \ldots\left(a_{i}-a_{h(m)}\right)} .
$$

We denote $\left(a_{i}-a_{0}\right) \ldots\left(a_{i}-a_{i-1}\right)\left(a_{i}-a_{i+1}\right) \ldots\left(a_{i}-a_{h(m)}\right)$ by $d(i)$. We notice that $\left|a_{i}\right| \leq 1$ for $i \in\{0, \ldots, h(m)\}$ and then

$$
\left|P_{m}(t)\right| \leq \frac{(h(m)+1)(1+|t|)^{h(m)} M^{m}}{\min _{i \in\{0, \ldots, h(m)\}} d(i)} .
$$

We have that $\left|a_{i}-a_{j}\right| \geq(|i-j| \epsilon) / h(m)$ because $\left|a_{i}-a_{j}\right| \geq|| a_{i}|-| a_{j}||$. This fact leads us to

$$
d_{i} \geq\left(\frac{\epsilon}{h(m)}\right)^{h(m)} i !(h(m)-i) !=\left(\frac{\epsilon}{h(m)}\right)^{h(m)} \frac{h(m) !}{\left(\begin{array}{c}
h(m) \\
i
\end{array}\right)} .
$$


The maximum of $\left(\begin{array}{c}h(m) \\ i\end{array}\right)(i \in\{0, \ldots, h(m)\})$ is $\left(\begin{array}{c}h(m) \\ {[h(m) / 2]}\end{array}\right)$ and then

$$
\left|P_{m}(t)\right| \leq(h(m)+1)(1+|t|)^{h(m)} M^{m} \frac{1}{\epsilon^{h(m)}}\left(\begin{array}{c}
h(m) \\
{[h(m) / 2]}
\end{array}\right) \frac{C e^{h(m)}}{\sqrt{2 \pi h(m)}} .
$$

for a $C>0$ by Stirling's formula. Since $\left(\begin{array}{c}h(m) \\ {[h(m) / 2]}\end{array}\right)<2^{h(m)}$ the root criterium proves that $\hat{f}(t, x)$ converges in a neighborhood of $x=0$, moreover $\hat{f}(t, x)$ converges in $D /(1+|t|)^{A}$ for some constant $D>0$.

The proof splits in two steps, to find uniform bounds in a "well distributed" set of points using Baire's theorem and then to estimate the coefficients of $\hat{f}$ using Lagrange's interpolation formula. The obstruction to convergence is located in the denominators of Lagrange's formula. Suppose we have a polynomial $P_{j}(t) \in \mathbb{C}[t]$ of degree $j-1$ and let us fix a compact set $K$. We choose $j$ points $a_{1}, \ldots, a_{j}$ in $K$ and we use them as nodes of interpolation. The geometric average of the denominators of Lagrange's interpolation formula is

$$
\left(\prod_{1 \leq k<l \leq j}\left|a_{k}-a_{l}\right|\right)^{\frac{2}{j}}
$$

We think of this number as an approximate lower bound for all denominators. We define the j-diameter of $K$

$$
\delta_{j}(K)=\sup _{\left(a_{1}, \ldots, a_{j}\right) \in K^{j}}\left(\prod_{1 \leq k<l \leq j}\left|a_{k}-a_{l}\right|\right)^{\frac{1}{\left(\begin{array}{c}
j \\
2
\end{array}\right)}} .
$$

The sequence $\delta_{j}(K)$ is decreasing [7] (p. 153) and the limit $\delta(K)=\lim _{j \rightarrow \infty} \delta_{j}(K)$ is called the transfinite diameter of $K$. We have:

Every $\hat{f} \in \mathbb{C}[t][[x]]_{A, B}$ converging on $\mathrm{K}$ is convergent $\Leftrightarrow \delta(K)>0$.

This result will not be proved explicitly. Anyway, the implication $\Rightarrow$ is a consequence of Theorem 6.1 whereas the implication $\Leftarrow$ is a consequence of Theorem 3.1. Those results are expressed in the language of potential theory. The connection between the approach in this section and the potential-theoretic one is made by

Theorem 2.1 (Fekete-Szëgo (see [7] p. 153)). Let $K \subset \mathbb{C}$ be a compact set, then

$$
\delta(K)=c(K) .
$$

The number $c(K)$ is by definition the logarithmic capacity of $K$. A set is polar if and only if its logarithmic capacity is 0 . 


\section{3. - Convergence on lines}

Next theorem is the main tool in this section.

Theorem 3.1. [5] Consider $\hat{f} \in \mathbb{C}\left[x_{1}, \ldots, x_{n}\right][[x]]_{A, B}$ and suppose that $\hat{f}$ converges on a non pluri-polar set $S\left(S \subset \mathbb{C}^{n}\right)$ of lines. Then $\hat{f}$ converges.

We refer the reader to [9] and [7] for definitions and applications in potential theory. The analogous theory in more than one complex variable is developed in [2].

Theorem 3.1 is a consequence of two results. These results are analogous to those used in Proposition 2.1. We will use that a countable union of Borel pluri-polar sets is pluri-polar playing the role of Baire's theorem, and BernsteinWalsh lemma instead of Lagrange's interpolation formula.

Lemma 3.1 (Bernstein-Walsh). (see [7] p. 156) If $\Delta \subset \mathbb{C}^{m}$ is not pluri-polar and $P$ is a polynomial of degree $d$, then we have for $z \in \mathbb{C}^{m}$ :

$$
|P(z)| \leq\|P\|_{C^{0}(\Delta)} e^{d V_{\Delta}(z)} .
$$

We define $\mathcal{L}$, the set of pluri-subharmonic functions defined in $\mathbb{C}^{m}$ of minimal growth i.e., $u(z)-\ln \|z\|$ is bounded above when $\|z\| \mapsto \infty$. Given a subset $\Delta \subset \mathbb{C}^{m}$, we define

$$
V_{\Delta}(z)=\sup \left\{u(z) / u \in \mathcal{L}, u_{\mid \Delta} \leq 0\right\} .
$$

The upper semi-continuous regularization $V_{\Delta}{ }^{*}$ of $V_{\Delta}$ is called the pluri-subharmonic Green function of $\Delta$. This function $V_{\Delta}{ }^{*}$ is either pluri-subharmonic or identically $+\infty$. We are in the former case when $\Delta$ is not pluri-polar.

The Bernstein-Walsh lemma approximates polynomials in terms of the function $V_{\Delta}$. Since $V_{\Delta}{ }^{*}$ belongs also to $\mathcal{L}$ the behavior of $V_{\Delta}{ }^{*}$ in a neighborhood of $\infty$ is quite simple. Based on this fact we present a corollary of the BernsteinWalsh lemma; it is a sort of version at $\infty$ similar to Nadirashvili's lemma. The corollary can be used instead of the lemma in order to prove Theorem 3.1.

COROLlary 3.1. If $\Delta \subset \mathbb{C}^{m}$ is not pluri-polar then there exist $R>0$ and $K>0$ such that if $P$ is a polynomial of any degree $d$ we have

$$
|P(z)| \leq\|P\|_{C^{0}(\Delta)} K^{d}\|z\|^{d} \quad \forall z \text { s.t. }\|z\| \geq R .
$$

We can now prove Theorem 3.1.

Proof of TheOrem 3.1. We develop $\hat{f}$ as a power series in the variable $x$

$$
\hat{f}=\sum_{j=0}^{\infty} P_{j}\left(x_{1}, \ldots, x_{n}\right) x^{j} .
$$


We consider the closed sets $A_{N}(N \in \mathbb{N})$ :

$$
A_{N}=\left\{\left(x_{1}, \ldots, x_{n}\right) \in \mathbb{C}^{n} /\left|P_{j}\left(x_{1}, \ldots, x_{n}\right)\right| \leq N^{j} \quad \forall j>0\right\} .
$$

The set $S$ is contained in $\cup_{N \in \mathbb{N}} A_{N}$. There exists $M \in \mathbb{N}$ such that $A_{M}$ is not pluri-polar because the countable union of Borel pluri-polar sets is pluri-polar. We have produced the uniform bounds in the set $A_{M}$.

We apply the Bernstein-Walsh lemma to the polynomials $P_{j}(j>0)$ and to the set $A_{M}$ :

$$
\left|P_{j}\left(x_{1}, \ldots, x_{n}\right)\right| \leq M^{j} e^{(A j+B) V_{A_{M}}\left(x_{1}, \ldots, x_{n}\right)} \leq M^{j} e^{(A j+B) V_{A_{M}}^{*}\left(x_{1}, \ldots, x_{n}\right)} .
$$

For each compact subset $K$ in $\mathbb{C}^{n}$ the function $V_{A_{M}}^{*}$ is bounded above by a constant $\sigma$ and in consequence:

$$
\left|P_{j}\left(x_{1}, \ldots, x_{n}\right)\right| \leq\left(M e^{A \sigma}\right)^{j} e^{B \sigma} \text { for }\left(x_{1}, \ldots, x_{n}\right) \in K \text { and } j>0 .
$$

The previous inequalities prove that $\hat{f}$ converges uniformly in a neighborhood of $K \times\{0\}$ towards a function $\tilde{f}$. Since $K$ can be any compact set the power series $\hat{f}$ defines a convergent function in a neighborhood of $x=0$.

REMARK 3.1. Keeping the notations in the previous proof, the set of lines of convergence of $\hat{f}$ is $\cup_{N \in \mathbb{N}} A_{N}$, which is a countable union of closed sets, i.e., a $F_{\sigma}$ set.

REMARK 3.2. Since the Green function belongs to $\mathcal{L}$ then there exist constants $C_{1} \in \mathbb{R}$ and $C_{2}>0$ such that $V_{A_{M}}^{*}<\log \left\|x_{1}, \ldots, x_{n}\right\|+C_{1}$ for $\left\|x_{1}, \ldots, x_{n}\right\|>C_{2}$. As a consequence a convergent element $f$ of $\mathbb{C}\left[x_{1}, \ldots, x_{n}\right][[x]]_{A, B}$ converges in

$$
|x|<\frac{C_{f}}{1+\left\|x_{1}, \ldots, x_{n}\right\|^{A}} \text { for some } C_{f}>0 .
$$

We have worked with elements of $\mathbb{C}\left[x_{1}, \ldots, x_{n}\right][[x]]_{A, B}$, lines invariant by $\frac{\partial}{\partial x}$ and convergence in a neighborhood of $x=0$. Since we are interested on properties of germs we will change the setup, we will consider formal power series or other formal objects, integral curves of the radial vector field $x_{1} \frac{\partial}{\partial x_{1}}+\ldots+x_{n} \frac{\partial}{\partial x_{n}}$ and convergence in the neighborhood of the origin. A series $\hat{f} \in \mathbb{C}\left[\left[x_{1}, \ldots, x_{n}\right]\right]$ converges in a line $\lambda\left(a_{1}, \ldots, a_{n}\right)(\lambda \in \mathbb{C})$ if the series $\hat{f}\left(a_{1} t, \ldots, a_{n} t\right)$ is convergent. Theorem 3.1 implies

Corollary 3.2. Let $\hat{f} \in \mathbb{C}\left[\left[x_{1}, \ldots, x_{n}\right]\right]$ be a formal power series. Suppose that $\hat{f}$ converges on a non pluri-polar of lines $\left(\right.$ in $\left.\mathbb{P}^{n-1}(\mathbb{C})\right)$ through the origin. Then $\hat{f}$ converges.

The proof is straightforward. We make a blow-up of the origin and we remark that the transformed of $\hat{f}$ restricted to each affine chart belongs to a ring isomorphic to $\mathbb{C}\left[y_{2}, \ldots, y_{n}\right]\left[\left[y_{1}\right]\right]_{1,0}$. 


\section{4. - Formal logarithmic functions}

We are going to extend Corollary 3.2 to more general objects than power series. These objects can be attached to geometrical ones in a natural way. We present a couple of examples related to diffeomorphisms.

Example. Consider a diffeomorphism of the form

$$
\varphi=\left(x_{1}(1+f), \ldots, x_{n}(1+f)\right), \quad f \in \mathbb{C}\left\{x_{1}, \ldots, x_{n}\right\} .
$$

All the lines through the origin are invariant by $\varphi$. If $f(0,0)=0$ then $j^{1} \varphi=I d$ and there exists a formal vector field $\widehat{X}$ such that $\varphi=\exp (\widehat{X})$. We denote by $\varphi_{r}\left(r \in \mathbb{P}^{n-1}(\mathbb{C})\right)$ the 1-dimensional germ of diffeomorphism defined in the line through the origin $r$ by restriction of $\varphi$ and centered at 0 . We define $S$ as the set of lines $r \in \mathbb{P}^{n-1}(\mathbb{C})$ such that $\varphi_{r}$ is the exponential of a convergent vector field. Corollary 3.2 applied to the coefficients of $\widehat{X}$ proves that either $\widehat{X}$ is convergent or $S$ is pluri-polar. It is possible to precise the nature of $S$. We call $C_{\varphi}$ the set of $r \in \mathbb{P}^{n-1}(\mathbb{C})$ such that the order of contact of $\varphi_{r}$ with the identity is not minimum, this set is analytic. Moreover, we have $S \backslash C_{\varphi}$ is analytic in $\mathbb{P}^{n-1}(\mathbb{C}) \backslash C_{\varphi}$; the proof is based on summability theory. The formal vector field $\widehat{X}$ can be summed in suitable domains [1]; the domains depend continuously on $r$ for $r \notin C_{\varphi}$. Then $\widehat{X}$ is convergent on $r$ if all the sums coincide by restriction to $r$. That provides analytic equations defining $S$.

As we have already seen the foliation supported by a logarithm of a germ of diffeomorphism can have a divergent meromorphic first integral. We present an example of logarithmic first integral. We remind the reader that a formal logarithmic function is an expression of the form $\hat{f}_{1}^{\lambda_{1}} \ldots \hat{f}_{p}^{\lambda_{p}}$ where $\hat{f_{i}} \in \mathbb{C}\left[\left[x_{1}, \ldots, x_{n}\right]\right]$ and $\lambda_{i} \in \mathbb{C}$ for all $i \in\{1, \ldots, p\}$. By definition $\hat{f}_{1}^{\lambda_{1}} \ldots \hat{f}_{p}^{\lambda_{p}}$ converges if there exist formal units $\hat{u}_{i}$ such that $\hat{u}_{i} \hat{f}_{i} \in \mathbb{C}\left\{x_{1}, \ldots, x_{n}\right\}$ for all $i \in\{1, \ldots, p\}$ and $\hat{u}_{1}^{\lambda_{1}} \ldots \hat{u}_{p}^{\lambda_{p}}$ converges.

Example. Consider the germ $\left(\lambda \notin \mathbb{R}^{-}\right)$

$$
\alpha=\left(\frac{x}{1-x}, y(1-x)^{\lambda}\right)=\exp \left(x^{2} \frac{\partial}{\partial x}-\lambda x y \frac{\partial}{\partial y}\right) .
$$

The logarithm of $\alpha$ supports the foliation $\Omega=(\lambda y d x+x d y=0)$. By construction the singularity of $\Omega$ at 0 is simple. We make a small perturbation of $\alpha$

$$
\varphi=\left(\frac{x}{1-x}, y(1-x)^{\lambda}+k(x)\right)=\exp \left(x\left(x \frac{\partial}{\partial x}-(\lambda y+\hat{h}) \frac{\partial}{\partial y}\right)\right),
$$

we choose $k(x) \in\left(x^{3}\right)$ because that implies $\hat{h} \in(x, y)^{2}$. The foliation $\hat{\Delta}$ supported by the logarithm of $\varphi$ has only two smooth formal integral curves, tangent to $x=0$ and $y=0$ respectively. The formal integral curve tangent to 
$x=0$ is $x=0$. We will show next that there exists $k(x) \in\left(x^{3}\right)$ such that the integral curve tangent to $y=0$ is divergent. The integral curve $y-\hat{g}(x)=0$ $(\hat{g} \in \mathbb{C}[[x]])$ satisfies

$$
(y-\hat{g}(x)) \circ \varphi \circ(x, \hat{g}(x))=0 .
$$

This equation is equivalent to

$$
\hat{g}\left(\frac{x}{1-x}\right)=\hat{g}(x)(1-x)^{\lambda}+k(x) .
$$

We choose for $x /(1-x)$ petals $P^{+}$and $P^{-}$, attractive and repulsive respectively, such that $(1-x)^{\lambda}$ is a unit in both of them and $P^{+} \cap P^{-}$is connected. Consider $x_{0} \in P^{+} \cap P^{-}$and $k(x)=x^{3} /\left(x-x_{0}\right)$. A possible choice is $x_{0}=i$. If $\hat{g}(x)$ is convergent then $\hat{g}$ admits two convergent extensions $g_{+}$and $g_{-}$to $P^{+}$and $P_{-}$respectively. The extensions are obtained by iteration using equation 1 . It's easy to prove that $g_{-}$is holomorphic in a neighborhood of $x_{0}$ and that $g_{+}$has a pole of order one in $x_{0}$. But that's not possible because $g_{+}=g_{-}=\hat{g}$ in a neighborhood of 0 and $P^{+} \cap P^{-}$is connected. We deduce that the the formal integral curve of $\widehat{\Delta}$ tangent to $y=0$ is divergent. The formal function $x^{\lambda} y$ is a first integral of $\Omega \equiv\left[d\left(x^{\lambda} y\right) / x^{\lambda-1}=0\right]$; since $\widehat{\Delta}$ is formally conjugated to $\Omega$ then the formal foliation $\widehat{\Delta}$ has a first integral of the form $x^{\lambda} \hat{y}(\widehat{\Delta} \equiv$ $\left.\left[d\left(x^{\lambda} \hat{y}\right) / x^{\lambda-1}=0\right]\right)$ where $\hat{y}=0$ is a divergent smooth formal curve tangent to $y=0$.

We will prove now the analogue of Corollary 3.2 for formal logarithmic functions. We will denote $\pi$ the application of blow-up of the origin.

PROPOSITION 4.1. Let $\hat{f}=\hat{f}_{1}^{\lambda_{1}} \ldots \hat{f}_{p}^{\lambda_{p}}$ be a formal logarithmic function and suppose that $\hat{f}$ converges on a non pluri-polar set of lines through the origin. Then $\hat{f}$ converges.

Proof. We will make the proof in three steps:

(1) We will make a blow-up of the origin and we will prove that $\hat{f}$ converges in a neighborhood of the exceptional divisor minus an analytic set.

(2) We will show that the result in dimension 2 implies the result in any dimension because of the Weierstrass preparation theorem.

(3) We will show the theorem in dimension 2.

First step. We call $S_{\hat{f}}$ the set of lines in which $\hat{f}$ converges. Let $v_{k}$ be the order of $\hat{f}_{k}$. The series $\hat{f}_{k}$ can be written in the form

$$
\hat{f}_{k}=f_{k, v_{k}}+f_{k, v_{k}+1}+\ldots
$$

where $f_{k, j}$ are homogeneous polynomials of degree $j$ and $f_{k, v_{k}} \neq 0$. We make a blow-up of the origin and take coordinates in the first chart. We have

$$
\hat{f}\left(y_{1}, y_{1} y_{2}, \ldots, y_{1} y_{n}\right)=\hat{f}_{1}\left(y_{1}, y_{1} y_{2}, \ldots, y_{1} y_{n}\right)^{\lambda_{1}} \ldots \hat{f}_{p}\left(y_{1}, y_{1} y_{2}, \ldots, y_{1} y_{n}\right)^{\lambda_{p}} .
$$


We define the analytic set $E=\left(f_{1, v_{1}}=0\right) \cup \ldots \cup\left(f_{p, v_{p}}=0\right)$; the set $E$ is a union of lines through the origin. We make a new parametrization of the lines through the origin which are not contained in $E$ by doing a ramification in $E$. In coordinates we have

$$
\begin{gathered}
y_{1}=w_{1} f_{1, v_{1}}\left(1, w_{2}, \ldots, w_{n}\right) \ldots f_{p, v_{p}}\left(1, w_{2}, \ldots, w_{n}\right) \\
y_{2}=w_{2} \\
\vdots \\
y_{n}=w_{n} .
\end{gathered}
$$

The function $\hat{f}_{1}$ is transformed into the function

$$
w_{1}{ }^{\nu_{1}} f_{1, v_{1}}{ }^{\nu_{1}+1} f_{2, v_{2}}{ }^{\nu_{1}} \ldots f_{p, v_{p}}{ }^{\nu_{1}}+\ldots+w_{1}{ }^{j} f_{1, v_{1}}{ }^{j} f_{2, v_{2}}{ }^{j} \ldots f_{p, v_{p}}{ }^{j} f_{1, j}+\ldots
$$

We can divide this expansion by $w_{1}{ }^{v_{1}} f_{1, v_{1}}{ }^{{ }^{1}+1} f_{2, v_{2}}{ }^{{ }_{1}} \ldots f_{p, v_{p}}{ }^{\nu_{1}}$ and we obtain

$$
1+a_{1}^{1}\left(w_{2}, \ldots, w_{n}\right) w_{1}+a_{2}^{1}\left(w_{2}, \ldots, w_{n}\right) w_{1}^{2}+\ldots
$$

where $a_{j}^{1}\left(w_{2}, \ldots, w_{n}\right)$ is a polynomial of degree at most $\left(v_{1}+\ldots+v_{p}+1\right) j$ for all $j \in \mathbb{N}$. The degree grows linearly with $j$. Repeating the same process with all the $\hat{f}_{k}(k \in\{1, \ldots, p\})$ we see that the convergence of $\hat{f}$ in a non pluri-polar set of lines implies the same property for

$$
H \stackrel{\text { def }}{=} \exp \left(\lambda_{1} \ln \left(1+\sum_{j=1}^{\infty} a_{j}^{1} w_{1}^{j}\right)+\ldots+\lambda_{p} \ln \left(1+\sum_{j=1}^{\infty} a_{j}^{p} w_{1}^{j}\right)\right) .
$$

Developing the right side of the previous equality we write $H$ in the form $\sum_{j=0}^{\infty} h_{j}\left(w_{2}, \ldots, w_{n}\right) w_{1}{ }^{j}$. By using the power series expansions of $\ln (1+x)$ and $\exp (x)$ in a neighborhood of 0 we deduce that $h_{j}$ is a polynomial of degree at most $\left(v_{1}+\ldots+v_{p}+1\right) j$ for all $j \in \mathbb{N}$.

The set $E \cup\left(x_{1}=0\right)$ is pluri-polar and then $S_{\hat{f}} \backslash\left(E \cup\left(x_{1}=0\right)\right)$ is not pluri-polar. We deduce that $H$ is convergent (Theorem 3.1) and then $\hat{f}$ is convergent in the neighborhood of $\pi^{-1}(0) \backslash\left(E \cup\left(x_{1}=0\right)\right)$. By changing the chart we obtain that $\hat{f}$ is convergent in the neighborhood of $\pi^{-1}(0) \backslash E$.

Second step. Suppose we know how to prove the proposition when $n=2$. Up to a linear change of coordinates we can write $\hat{f}_{1}$ in the form

$$
\hat{f}_{1}=\hat{u}\left(x_{1}, \ldots, x_{n}\right)\left(x_{n}{ }^{m}+\hat{a}_{m-1} x_{n}{ }^{m-1}+\ldots+\hat{a}_{0}\right)
$$

where $m$ is minimal, $\hat{u}$ is a formal unit and all the $\hat{a}_{j}(j \in\{0, \ldots, m-1\})$ belong to $\mathbb{C}\left[\left[x_{1}, \ldots, x_{n-1}\right]\right]$. This is the formal version of Weierstrass preparation theorem. We denote $p r:\left(x_{1}, \ldots, x_{n}\right) \mapsto\left(x_{1}, \ldots, x_{n-1}\right)$. We consider the set 
$P_{n}$ of vectorial 2-planes in $\mathbb{C}^{n}$ containing the line $\left(x_{1}=0\right) \cap \ldots \cap\left(x_{n-1}=0\right)$. The projection $p r$ establishes a bijection between the set of such 2-planes and the lines through the origin in $\left(x_{1}, \ldots, x_{n-1}\right)$ (which are parameterized by $\mathbb{P}^{n-2}(\mathbb{C})$ ). For any 2-plane $P \in P_{n}$ except maybe a proper analytic set $A_{n} \subset \mathbb{P}^{n-2}(\mathbb{C})$ the series $\hat{f}$ converges on all the lines through the origin contained in $P$ except maybe a finite set. Since the proposition is true in dimension 2 then $\hat{f}_{1 \mid P}=\hat{u}_{1, P} f_{1, P}$ where $\hat{u}_{1, P}$ is a formal unit in $P$ and $f_{1, P}$ is a convergent germ on $P$. Applying Weierstrass preparation theorem to $\hat{f}_{1, P}$ is the same that applying the preparation theorem to $\hat{f}_{1}$ and then to make the restriction to $P$. We deduce that the series $\hat{a}_{i}(i \in\{0, \ldots, m-1\})$ converges on $\operatorname{pr}(P)$. Since $\operatorname{pr}\left(P_{n} \backslash A_{n}\right)$ is the complementary of an analytic set of lines and then non pluri-polar we deduce that $\hat{a}_{i} \in \mathbb{C}\left\{x_{1}, \ldots, x_{n-1}\right\}$ for all $i \in\{0, \ldots, m-1\}$ (Corollary 3.2). We have $\hat{f}_{i}=\hat{u}_{i} f_{i}$ for all $i \in\{0, \ldots, m-1\}\left(\hat{u}_{i}\right.$ is a formal unit and $f_{i}$ is convergent). The result is proved by applying Corollary 3.2 to $\hat{u}_{1}^{\lambda_{1}} \ldots \hat{u}_{p}^{\lambda_{p}}$.

Third step. We can make several blow-ups in order to simplify the formal curve $\hat{f}_{1} \ldots \hat{f}_{p}=0$. We denote $\pi_{r}$ the composition of all the blow-ups. We ask $\pi_{r}$ to be a desingularization of $\hat{f}_{1} \ldots \hat{f}_{p}=0$. Moreover, we choose $\pi_{r}$ such that the strict transforms of $\hat{f}_{j}=0$ and $\hat{f}_{k}=0$ pass through different points $P_{j}$ and $P_{k}$ respectively of the exceptional divisor $\pi_{r}^{-1}(0)$ if $j \neq k$. Using the method of the first step we want to see that $\hat{f} \circ \pi_{r}$ converges in a neighborhood of $\pi_{r}{ }^{-1}(0)$. We will call $D$ the component of $\pi_{r}{ }^{-1}(0)$ corresponding to the first blow-up. As in the first step the function $\hat{f} \circ \pi_{r}$ converges in a neighborhood of $D$ except a finite set $F$. The set $F$ contains two types of points, namely intersections of irreducible components of $\pi_{r}{ }^{-1}(0)$ with $D$ (corners) and points of $D$ contained in the strict transform of any $\hat{f}_{k}=0(1 \leq k \leq p)$.

Let us consider a component $D_{1}$ of $\pi_{r}{ }^{-1}(0)$ and a finite subset $F_{1} \subset D_{1}$. We will prove that if $\hat{f} \circ \pi_{r}$ converges in a neighborhood of $D_{1} \backslash F_{1}$ then $\hat{f} \circ \pi_{r}$ converges in a neighborhood of $D_{1}$. Suppose this result is true, then $\hat{f} \circ \pi_{r}$ will be convergent in a neighborhood of $D$. First step provides convergence in the neighborhood of $D^{\prime} \backslash F^{\prime}$ where $F^{\prime} \subset D^{\prime}$ is a finite set and $D^{\prime}$ is any component of $\pi_{r}{ }^{-1}(0)$ such that $D^{\prime} \cap D \neq \emptyset$. By making $D_{1}=D^{\prime}$ we obtain convergence of $\hat{f} \circ \pi_{r}$ in a neighborhood of $D^{\prime}$. Convergence of $\hat{f} \circ \pi_{r}$ in a neighborhood of $\pi_{r}{ }^{-1}(0)$ is proved by induction.

We choose an affine chart $(a, b)$ centered in a point in $F_{1}$. We can suppose that $a=0$ is the local equation of $D_{1}$. There exists a neighborhood $V \subset(a=0)$ of $(0,0)$ such that $\hat{f} \circ \pi_{r}$ converges in a neighborhood of $V \backslash\{0\}$. We can assume that if $(0,0)$ is a corner then $b=0$ is the equation of the other component of $\pi_{r}{ }^{-1}(0)$ passing through $(a, b)=(0,0)$. If there exists (it's always unique) a strict transform of a $\hat{f}_{k}=0(k \in\{1, \ldots, p\})$ passing through $(a, b)=(0,0)$ we call it $\hat{g}=0$. The formal function $\hat{f} \circ \pi_{r}$ is of the form

$$
a^{\mu_{1}} b^{\mu_{2}} \hat{g}^{\mu_{3}} \hat{u}(a, b) \text { s.t. }\{\hat{g}, \hat{u}\} \subset \mathbb{C}[b][[a]]_{A, B} \text { for some } A \text { and } B \text {. }
$$


The function $\hat{f} \circ \pi_{r}$ converges in a neighborhood of $(a, b)=(0,0)$ because we can apply Theorem 3.1 to $\hat{u}$ if $\mu_{3}=0$ and to $\hat{g} \hat{u}^{1 / \mu_{3}}$ if $\mu_{3} \neq 0$. Convergence in a neighborhood of $D_{1}$ is now proved.

Since $\hat{f} \circ \pi_{r}$ converges in a neighborhood of $\pi_{r}{ }^{-1}(0)$ then $\hat{f}_{j} \circ \pi_{r}$ defines a convergent curve in the neighborhood of $P_{j}$. This curve defines an analytic curve $\gamma(s)$ in the neighborhood of 0 by undoing the blow-ups. The ideal of formal power series $\hat{d}$ such that $\hat{d} \circ \gamma=0$ is prime, principal and has a convergent generator $f_{j}$ (by the Weierstrass division theorem). We have $\hat{f}_{j}=\hat{u}_{j} f_{j}$ for some formal unit $\hat{u}_{j}$ and all $1 \leq j \leq p$. The end of the proof is like in the second step.

Remark 4.1. The Proposition 4.1 is true for formal meromorphic functions because meromorphic implies logarithmic.

\section{5. - Changing the fibration}

A germ of foliation is dicritical if it supports infinitely many invariant curves. The radial fibration is the easiest dicritical foliation, anyway in dimension 2 its role can be played by any other dicritical foliation. We replace convergence on lines through the origin by convergence on integral curves of the foliation. By definition a formal logarithmic function $\hat{f}$ converges on a curve $\Gamma$ if $\hat{f} \circ \gamma$ converges for a Puiseux parametrization $\gamma$ of $\Gamma$.

Proposition 5.1. Let $\hat{f}=\hat{f}_{1}^{\lambda_{1}} \ldots \hat{f}_{p}^{\lambda_{p}}$ be a formal logarithmic function and $\Omega$ a dicritical germ of foliation. If $\hat{f}$ converges on a non pluri-polar set of integral curves of $\Omega$ then it does converge.

Proof. We know that $\Omega$ admits a desingularization (making blow-ups of points) with a finite number of dicritical components [8]. All the integral curves except a finite number cut one of these dicritical components. There exists a dicritical component $C_{0} \sim \mathbb{P}^{1}(\mathbb{C})$ and a non pluri-polar set $S_{0} \subset C_{0}$ such that $\Omega$ is transversal to $C_{0}$ on $S_{0}$ and $\hat{f}$ converges on restriction to the leaves of $\Omega$ passing through points of $S_{0}$. This is a consequence of the definition of convergence on non pluri-polar sets of integral curves.

We notice that we can reduce the problem to the case where $C_{0}$ is the divisor of the first blow-up. In general there is an application $\mu$ which is a finite composition of blow-ups $\mu_{1}, \ldots, \mu_{r}$ of $(0,0), \ldots, P_{r-1} \in\left(\mu_{1} \circ \ldots \circ \mu_{r-1}\right)^{-1}(0)$ respectively. Then

$$
\hat{f} \circ \mu_{1} \circ \ldots \circ \mu_{r-1}
$$

is convergent in a neighborhood of $P_{r-1}$ because we can undo the last blow-up $\mu_{r}$ by taking $C_{0}=\mu_{r}^{-1}\left(P_{r-1}\right)$. Convergence of $\hat{f}$ in the neighborhood of $P_{r-2}$, $\ldots,(0,0)$ is obtained by an iterative application of Proposition 4.1. 
From now on we suppose that $C_{0}=\pi^{-1}(0)$. Our aim is transforming $\Omega$ in the radial fibration. The radial fibration is always transversal to $\pi^{-1}(0)$ whereas $\Omega$ can have singularities and tangencies. We are going to make $\Omega$ transversal to $\pi^{-1}(0)$ by doing a ramification in all the places where $\Omega$ is not transversal to $\pi^{-1}(0)$. This set is finite, we denote it by $\left\{Q_{1}, \ldots, Q_{s}\right\}$; we can suppose that it's contained in the first chart of the blow-up of $0(x=x$, $y=x t$ ). The foliation $\pi^{*} \Omega$ is given by the 1 -form

$$
A(x, t) d x+\left(B_{1}(x, t) x+B_{0}(t)\right) d t .
$$

The polynomial $B_{0}(t)$ is not identically 0 because $\Omega$ is generically transversal to $\pi^{-1}(0)$. The set $\left\{Q_{1}, \ldots, Q_{s}\right\}$ is the set of zeros of $B_{0}(t)$. If $d=\operatorname{deg}\left(B_{0}\right)$ we consider the ramification $\sigma$ in coordinates $(x, y)$ :

$$
\sigma=\left\{\begin{array}{l}
x=x\left[x^{d} B_{0}(y / x)\right]^{2} \\
y=y\left[x^{d} B_{0}(y / x)\right]^{2} .
\end{array}\right.
$$

The foliation $\pi^{*} \sigma^{*} \Omega$ is transversal to $\pi^{-1}(0)$ except maybe in a subset of $\left\{Q_{1}, \ldots, Q_{s}\right\}$. In coordinates $(x, t)$ we have $\sigma(x, t)=\left(x^{2 d+1} B_{0}(t)^{2}, t\right)$, as a consequence $\pi^{*} \sigma^{*} \Omega=(\sigma \circ \pi)^{*} \Omega$ is generically colinear to

$$
(A \circ \sigma)\left(2 x^{2 d+1} d\left(B_{0}(t)\right)+B_{0}(t) d\left(x^{2 d+1}\right)\right)+\left(\left(B_{1} \circ \sigma\right) x^{2 d+1} B_{0}(t)+1\right) d t,
$$

which is always transversal to $x=0$. A theorem of Poincare shows that there exists a diffeomorphism $\varphi \in \operatorname{Diff}\left(\mathbb{C}^{2}, 0\right)$ which conjugates the radial fibration and $\sigma^{*} \Omega$. The lines through the origin are transformed in leaves of $\Omega$ by $\sigma \circ \varphi$. The value of $\hat{f} \circ \sigma \circ \varphi$ restricted to a line $l$ is a ramification around the origin of the restriction of $\hat{f}$ to $(\sigma \circ \varphi)(l)$. The function $\hat{f} \circ \sigma \circ \varphi$ is convergent by Proposition 4.1 and then $\hat{f} \circ \sigma$ is also convergent; the function $\hat{f} \circ \pi$ is convergent in a neighborhood of $\pi^{-1}(0) \backslash\left\{Q_{1}, \ldots, Q_{s}\right\}$. Proposition 4.1 proves the convergence of $\hat{f}$.

REMARK 5.1. The same result is true for formal power series and formal meromorphic functions because both are particular cases of formal logarithmic functions.

\section{6. - Optimality of the results}

We can be much more precise if the parameter space is 1-dimensional. Next theorem characterizes any subset of $\mathbb{C}$ which is the set of lines of convergence of an element of $\mathbb{C}[t][[x]]_{A, B}$.

THEOREM 6.1. Let $E$ be a subset of $\mathbb{C}$, then there exists a divergent element of $\mathbb{C}[t][[x]]_{A, B}$ converging on the lines in $E$ and diverging on the lines in $\mathbb{C} \backslash E$ if and only if $E$ is a $F_{\sigma}$ polar set. 
We introduce the Harnack distance for a domain in $\mathbb{P}^{1}(\mathbb{C})$; it is a key tool of the proof.

Definition: Let $D$ be a domain in $\mathbb{P}^{1}(\mathbb{C})$. Given $z, w \in D$, the Harnack distance between $z$ and $w$ is the smallest number $\tau_{D}(z, w)$ such that, for every positive harmonic function $\mathrm{h}$ on $D$,

$$
\tau_{D}(z, w)^{-1} h(w) \leq h(z) \leq \tau_{D}(z, w) h(w)
$$

The main properties of the Harnack distance are (see [7] p. 14):

- (Subordination principle) Let $f: D_{1} \rightarrow D_{2}$ be a holomorphic map between domains $D_{1}$ and $D_{2}$ in $\mathbb{P}^{1}(\mathbb{C})$. Then

$$
\tau_{D_{2}}(f(z), f(w)) \leq \tau_{D_{1}}(z, w) \quad\left(z, w \in D_{1}\right)
$$

with equality if $f$ is a conformal mapping of $D_{1}$ onto $D_{2}$.

- If $D_{1} \subset D_{2}$ then $\tau_{D_{2}}(z, w) \leq \tau_{D_{1}}(z, w)\left(z, w \in D_{1}\right)$. This is trivially deduced from the subordination principle by taking $f$ equal to the inclusion map.

- If $D$ is a domain of $\mathbb{P}^{1}(\mathbb{C})$, then $\log \tau_{D}$ is a continuous semimetric on $D$. In particular $\tau_{D}$ is a continuous function.

Proof of Theorem 6.1. The implication $\Rightarrow$ is already proved (Theorem 3.1 and Remark 3.1).

$(\Leftarrow)$ We can suppose $A=1$ and $B=0$. If there exists $\hat{f}=\sum_{j=0}^{\infty} P_{n}(t) x^{n} \in$ $\mathbb{C}[t][[x]]_{1,0}$ converging on the lines in $E$ and diverging elsewhere then we consider $f(x, t) \circ\left(x^{d}, t\right)$ for some $d \in \mathbb{N}$. This series converges on the same lines than $\hat{f}$ and it belongs to $\mathbb{C}[x][[t]]_{A, B}$ for $d \geq A^{-1}$.

If $E=\emptyset$ then $\hat{f}=\sum_{n=0}^{\infty} n ! x^{n}$ does not converge on any line. We suppose from now on that $E$ is a non-empty $F_{\sigma}$ polar set. There exists a family of non-empty compact polar sets $\left\{K_{j}\right\}_{j \in \mathbb{N}}$, where $K_{j} \subset K_{j+1}$ for all $j \in \mathbb{N}$, such that $E=\cup_{j \in \mathbb{N}} K_{j}$. We want to express $E$ as a countable union of disjoint compact polar sets. Since

$$
E=K_{1} \cup\left(K_{2} \backslash K_{1}\right) \cup\left(K_{3} \backslash K_{2}\right) \cup \ldots
$$

it is enough to write $K_{j+1} \backslash K_{j}(j>1)$ as a countable disjoint union of compact polar sets.

We define over $K_{j+1}(j>1)$ the function $T(t)=d\left(t, K_{j}\right)$. The function $T$ is continuous, proper and such that $T^{-1}(0)=K_{j}$. We have

$$
|T(x)-T(y)|=\left|d\left(x, K_{j}\right)-d\left(y, K_{j}\right)\right| \leq d(x, y)=|x-y|
$$

and in consequence (cf. [7] p. 137) $c\left(T\left(K_{j+1}\right)\right) \leq c\left(K_{j+1}\right)=0$. The set $T\left(K_{j+1}\right) \subset \mathbb{R}$ is a polar set and then totally disconnected. There exists a strictly 
decreasing sequence $\left\{\lambda_{n}\right\}_{n \in \mathbb{N}}$ of real positive numbers such that $\lim _{n \rightarrow \infty} \lambda_{n}=0$ and $\lambda_{n} \notin T\left(K_{j+1}\right)$ for all $n>0$. The sets

$$
T^{-1}\left[\lambda_{1}, \infty\right), T^{-1}\left[\lambda_{2}, \lambda_{1}\right], T^{-1}\left[\lambda_{3}, \lambda_{2}\right], \ldots
$$

are compact, different ones are disjoint and their union is $K_{j+1} \backslash K_{j}$.

The set $E$ is equal to $\cup_{j \in \mathbb{N}} M_{j}$ where for all $j>0$ the set $M_{j}$ is compact and polar and $M_{j} \cap M_{k}=\emptyset$ if $j \neq k$. We can suppose that $M_{1} \neq \emptyset$. For any subset $D \subset \mathbb{C}$ and $\lambda \in \mathbb{R}^{+}$we denote $\cup_{d \in D} B(d, \lambda)$ by $V_{\lambda}(D)$. The next step of the proof is finding a sequence $\left\{\mu_{j}\right\}$ of strictly positive real numbers such that for any $t_{0} \in \mathbb{C} \backslash E$ the sequence $\left\{S_{j}\right\}_{j \in \mathbb{N}}$ such that

$$
S_{2 k-1}=V_{\mu_{2 k-1}}\left(M_{1} \cup \ldots \cup M_{k}\right), \quad S_{2 k}=V_{\mu_{2 k}}\left(M_{1} \cup \ldots \cup M_{k}\right)
$$

has infinitely many elements not containing $t_{0}$. We suppose that such a sequence exists. We will make the proof at the end.

We fix $t_{0} \in M_{1}$ and $j \in \mathbb{N}$. If $\epsilon>0$ is small enough then the segment $\left[t_{0}, t_{0}+\epsilon\right]$ is contained in $S_{j}$. The set

$$
D_{j, \epsilon}=\mathbb{P}^{1}(\mathbb{C}) \backslash\left(\left[t_{0}, t_{0}+\epsilon\right] \cup M_{1} \cup \ldots \cup M_{\left[\frac{j+1}{2}\right]}\right)
$$

is connected because $\mathbb{P}^{1}(\mathbb{C}) \backslash\left[t_{0}, t_{0}+\epsilon\right]$ is connected and the $M_{k}(k>0)$ are all polar. We denote $F_{j, \epsilon}$ the compact set $\mathbb{P}^{1}(\mathbb{C}) \backslash D_{j, \epsilon}$. The Bernstein's lemma (see [7] p. 156) shows that

$$
\left(\frac{\left|q_{j}(t)\right|}{\left\|q_{j}\right\|_{F_{j, \epsilon}}}\right)^{1 / d_{j}} \geq e^{\inf _{z \in \mathbb{C} \backslash S_{j}} V_{F_{j, \epsilon}^{*}}^{*}(z)}\left(\frac{c\left(F_{j, \epsilon}\right)}{\delta_{d_{j}}\left(F_{j, \epsilon}\right)}\right)^{\sup _{z \in \mathbb{C} \backslash S_{j}} \tau_{D_{j, \epsilon}}(z, \infty)}
$$

for any Fekete polynomial $q_{j}$ associated to $F_{j, \epsilon}$ of degree $d_{j}$ and any $t \in \mathbb{C} \backslash S_{j}$. We remind that $V_{F_{j, \epsilon}}^{*}$ is the Green function associated to $F_{j, \epsilon}$. The value $\delta_{j}\left(F_{j, \epsilon}\right)$ $(j>1)$ is the $j$-diameter associated to the compact set $F_{j, \epsilon}$ (cf. [7] p. 152). We choose $\epsilon_{0}$ such that $\left[t_{0}, t_{0}+\epsilon_{0}\right] \subset S_{j}$. The application $h_{\epsilon_{0}, \epsilon}=t \mapsto\left(t-t_{0}\right) \epsilon / \epsilon_{0}+t_{0}$ is a biholomorphism from $\mathbb{P}^{1}(\mathbb{C}) \backslash\left[t_{0}, t_{0}+\epsilon_{0}\right]$ to $\mathbb{P}^{1}(\mathbb{C}) \backslash\left[t_{0}, t_{0}+\epsilon\right]$. Using the invariance of the Green function

$$
V_{F_{j, \epsilon}}^{*}\left(h_{\epsilon_{0}, \epsilon}(t)\right)=V_{F_{j, \epsilon_{0}}^{*}}^{*}(t),
$$

we realize that

$$
\inf _{t \in \mathbb{C} \backslash S_{j}} V_{F_{j, \epsilon}}^{*}(t)=\inf _{t \in h_{\epsilon_{0}, \epsilon}^{-1}\left(\mathbb{C} \backslash S_{j}\right)} V_{F_{j, \epsilon_{0}}}^{*}(t) .
$$

The functions $h_{\epsilon_{0}, \epsilon}^{-1}$ tend uniformly in $\mathbb{C} \backslash S_{j}$ to $\infty$ when $\epsilon$ tends to 0 , and then

$$
\inf _{t \in \mathbb{C} \backslash S_{j}} V_{F_{j, \epsilon}}^{*}(t) \rightarrow \infty \text { when } \epsilon \rightarrow 0 .
$$


We have the inclusion $D_{j, \epsilon_{0}} \subset D_{j, \epsilon}$ if $\epsilon<\epsilon_{0}$ and then $\tau_{D_{j, \epsilon_{0}}}(t, \infty) \geq \tau_{D_{j, \epsilon}}(t, \infty)$ for all $t \in \mathbb{P}^{1}(\mathbb{C}) \backslash S_{j}$. The set $\mathbb{P}^{1}(\mathbb{C}) \backslash S_{j}$ is a compact set contained in $D_{j, \epsilon_{0}}$. The continuity of $\tau_{D_{j, \epsilon_{0}}}$ implies the existence of $M>0$ such that $\tau_{D_{j, \epsilon_{0}}}(t, \infty)<M$ for all $t \in \mathbb{P}^{1}(\mathbb{C}) \backslash S_{j}$. Then $\tau_{D_{j, \epsilon}}(t, \infty)<M$ for all $t \in \mathbb{C} \backslash S_{j}$ and all $\epsilon \leq \epsilon_{0}$. By the Fekete-Szëgo theorem (cf. [7] p. 153) we have

$$
\frac{c\left(F_{j, \epsilon}\right)}{\delta_{d_{j}}\left(F_{j, \epsilon}\right)} \rightarrow 1, \quad \text { when } \quad \lim _{j \rightarrow \infty} d_{j}=\infty
$$

for any $\epsilon \leq \epsilon_{0}$.

We choose $\epsilon \leq \epsilon_{0}$ such that $\inf _{t \in \mathbb{C} \backslash S_{j}} V_{F_{j, \epsilon}}^{*}(t) \geq K$ and $e^{K} \geq 2 j$. We can choose now $d_{j}>d_{j-1}$ such that

$$
\frac{c\left(F_{j, \epsilon}\right)}{\delta_{d_{j}}\left(F_{j, \epsilon}\right)} \geq S<1 \quad \text { and } \quad S^{M} \geq 1 / 2 .
$$

All the inequalities put together give to us

$$
\left(\frac{\left|q_{j}(t)\right|}{\left\|q_{j}\right\|_{F_{j, \epsilon}}}\right)^{1 / d_{j}} \geq j, \quad \forall t \in \mathbb{C} \backslash S_{j} .
$$

Let $p_{j}=q_{j} /\left\|q_{j}\right\|_{F_{j, \epsilon}}$. We define $\hat{f}=\sum_{j=1}^{\infty} p_{j}(t) x^{d_{j}}$. The series $\hat{f}$ converges on any $t_{0} \in E$ with radius of convergence at least 1 , because $\left|p_{j}\left(t_{0}\right)\right|^{1 / d_{j}} \leq 1$ for all $j$ big enough. If $t_{0} \notin E$ there exists a strictly increasing sequence $\left\{s_{k}\right\}_{k \in \mathbb{N}}$ such that $t_{0}$ does not belong to $S_{s_{k}}$ for all $k \in \mathbb{N}$. We have

$$
\left|p_{s_{k}}\left(t_{0}\right)\right|^{1 / d_{s_{k}}} \geq s_{k} \rightarrow \infty
$$

and then $\hat{f}$ does not converge on $t_{0}$.

Let us construct the sequence $\left\{S_{j}\right\}_{j \in \mathbb{N}}$. Let $\mu_{1}$ be any positive real number. Since $M_{2} \cap M_{1}=\emptyset$ we choose $\mu_{2}$ smaller than $d\left(M_{1}, M_{2}\right) / 2$. Suppose the first $k-1$ terms of the sequence $\left\{\mu_{i}\right\}$ are given. If $k$ is even then we select $\mu_{k}$ such that each two of the sets

$$
\overline{V_{\mu_{k}}\left(M_{1}\right)}, \ldots, \overline{V_{\mu_{k}}\left(M_{\frac{k}{2}+1}\right)}
$$

are disjoint and $\mu_{k} \leq \mu_{k-1} / 2$. If $k$ is odd then we define $\mu_{k}=\mu_{k-1}$. By definition we have $\lim _{k \rightarrow \infty} \mu_{k}=0$. It is enough to prove $\left(\cap_{j \geq n_{0}} S_{j}\right) \subset E$ for any $n_{0} \in \mathbb{N}$; we will show that

$$
\cap_{j=n_{0}}^{n_{0}+l} S_{j}=V_{\mu_{n_{0}+l}}\left(M_{1} \cup \ldots \cup M_{\left[\frac{n_{0}+1}{2}\right]}\right) .
$$


Last equality implies $\cap_{j=n_{0}}^{\infty} S_{j}=M_{1} \cup \ldots \cup M_{\left[\left(n_{0}+1\right) / 2\right]} \subset E$.

We will prove $\cap_{j=n_{0}}^{n_{0}+l} S_{j}=V_{\mu_{n_{0}+l}}\left(M_{1} \cup \ldots \cup M_{\left[\left(n_{0}+1\right) / 2\right]}\right)$ by induction on $l$, it is true for $l=0$. Suppose it is true for $l=p-1$, we have

$$
\cap_{j=n_{0}}^{n_{0}+p} S_{j}=\cap_{j=n_{0}}^{n_{0}+p-1} S_{j} \cap S_{n_{0}+p-1} \cap S_{n_{0}+p} .
$$

All our choices imply that $\overline{V_{\mu_{n_{0}+p-1}}\left(M_{j}\right)} \cap \overline{V_{\mu_{n_{0}+p-1}}\left(M_{k}\right)}=\emptyset$ if $j \neq k$ and $\{j, k\} \subset\left\{1, \ldots,\left[\frac{n_{0}+p+1}{2}\right]\right\}$. In consequence we have

$$
S_{n_{0}+p-1} \cap S_{n_{0}+p}=V_{\mu_{n_{0}+p}}\left(M_{1} \cup \ldots \cup M_{\left[\frac{n_{0}+p}{2}\right]}\right) .
$$

The set $\cap_{j=n_{0}}^{n_{0}+p} S_{j}$ is equal to

$$
V_{\mu_{n_{0}+p-1}}\left(M_{1} \cup \ldots \cup M_{\left[\left(n_{0}+1\right) / 2\right]}\right) \cap V_{\mu_{n_{0}+p}}\left(M_{1} \cup \ldots \cup M_{\left[\frac{n_{0}+p}{2}\right]}\right)
$$

and then

$$
\cap_{j=n_{0}}^{n_{0}+p} S_{j}=V_{\mu_{n_{0}+p}}\left(M_{1} \cup \ldots \cup M_{\left[\frac{n_{0}+1}{2}\right]}\right)
$$

using $\overline{V_{\mu_{n_{0}+p-1}}\left(M_{j}\right)} \cap \overline{V_{\mu_{n_{0}+p-1}}\left(M_{k}\right)}=\emptyset$ if $j<k \leq\left[\left(n_{0}+p+1\right) / 2\right]$.

The outer logarithmic capacity of a set $E \subset \mathbb{C}$ is defined as follows

$$
c^{*}(E)=\inf \{c(U) / U \text { is an open set such that } E \subset U\} .
$$

Since the $F_{\sigma}$ polar sets are the sets of convergence of the divergent series we are interested in

$$
c^{\sharp}(E)=\inf \left\{c(F) / F \text { is a } F_{\sigma} \text { and } E \subset F\right\} .
$$

Choquet's theorem implies that for all $E \subset \mathbb{C}$ both quantities $c^{*}(E)$ and $c^{\sharp}(E)$ coincide. We divide the sets of the plane in three types

Big The sets $E \subset \mathbb{C}$ such that $c^{*}(E)>0$

Small The sets $E \subset \mathbb{C}$ such that $c^{*}(E)=0$ and there exists a $F_{\sigma}$ polar set $F$ such that $E \subset F$

Mixed The sets $E \subset \mathbb{C}$ such that $c^{*}(E)=0$ but $c(F)>0$ for every $F_{\sigma}$ set $F$ containing $E$.

If a formal power series $\hat{f} \in \mathbb{C}[t][[x]]_{A, B}$ converges on the lines on a big or mixed set then $\hat{f}$ converges. This property is no longer true for small sets (Theorem 6.1).

The properties of the big and mixed sets are different. If $V$ is an open neighborhood of $x=0$ in $\mathbb{C}^{2}$ and $E$ is a subset of $\mathbb{C}$ we will say that a formal series $\hat{f} \in \mathbb{C}[t][[x]]_{A, B}$ converges on $E$ in $V$ if for any line $r \in E$ there exists an analytic function in $r \cap V$ whose power series development at the origin is $\hat{f}_{\mid r}$.

We define $G_{U}=\inf _{(t, x) \in \partial U}\left\{|x|\left(1+|t|^{A}\right)\right\}$ for any open neighborhood $U$ of $x=0$. Then 
Proposition 6.1. Let $E \subset \mathbb{C}$ be a big set. There exists a constant $L_{E}>0$ such that for any $V$ open neighborhood of $x=0$ all the series converging on $E$ in $V$ are analytic in some open neighborhood $W(V)$ of $x=0$ such that $G_{W(V)} / G_{V} \geq L_{E}$.

We must understand in the previous result that if $G_{V}=0$ then $G_{W(V)}>0$ and if $G_{V}=\infty$ then $W(V)=\mathbb{C}^{2}$.

Proposition 6.2. Let $E \subset \mathbb{C}$ be a mixed set. Let $V$ be an open neighborhood of $x=0$ such that $\sup _{(t, x) \in V}\left\{|x|\left(1+|t|^{A}\right)\right\}<+\infty$. Then for all W open neighborhood of $x=0$ there exists a formal series $\hat{f}_{W} \in \mathbb{C}[t][[x]]_{A, B}$ such that $\hat{f}_{W}$ converges on $E$ in $V$ but $\hat{f}_{W}$ does not converge in $W$.

The mixed sets share properties with both the big and the small sets. Every power series converging on $E$ is convergent (as in the big sets), but for any suitable open neighborhood $V$ of $x=0$ the intersection of the domains of convergence of the series converging on $E$ in $V$ is not a neighborhood of $x=0$ (as in the small sets).

Proof of 6.1. Let $\hat{f}=\sum_{i=0}^{\infty} P_{n}(t) x^{n} \in \mathbb{C}[t][[x]]_{A, B}$ a power series converging on $E$ in $V$. We define $h(n)=[A n+B]$; we have $\operatorname{deg} P_{n} \leq h(n)$ by definition. The series $\hat{f}$ converges in a neighborhood of $x=0$ by Theorem 3.1. We consider the sets $E_{R}=\{t \in E /|t|<R\}$. Since $c^{*}(E)>0$ then there exists $R \in \mathbb{N}$ such that $c^{*}\left(E_{R}\right)>0$. It's enough to prove the proposition for $E_{R}$. There exists a constant $C_{V} \geq 0$ such that

$$
\lim \sup _{n \rightarrow \infty}\left|P_{n}(t)\right|^{\frac{1}{n}} \leq C_{V} \quad \forall t \in E_{R} .
$$

Since $E_{R}$ is bounded we have $C_{V} \leq \kappa / G_{V}$ for some $\kappa>0$.

We claim that the functions $u_{n}=(1 / n) \log \left|P_{n}(t)\right|$ are uniformly bounded above over any compact set. This fact is proved in the proof of Theorem 3.1, it can be also be deduced directly using the convergence of $\hat{f}$. We define over $\mathbb{C}$ the real valued function

$$
u=\lim \sup _{n \rightarrow \infty} \frac{1}{n} \log \left|P_{n}(t)\right| .
$$

All the $u_{n}$ are uniformly locally bounded above and hence the Brelot-Cartan's theorem assures that $u^{*}$ (the upper semi-continuous regularization of $u$ ) is either subharmonic or identically equal to $-\infty$. Moreover, the functions $u$ and $u^{*}$ are equal outside of a Borel polar set. There exists a set $D \subset E_{R}$ such that $c^{*}(D)=c^{*}\left(E_{R}\right)$ and $u^{*}{ }_{\mid D}=u_{\mid D}$. The function $u^{*}$ is smaller than $\log C_{V}$ in $D$. Semi-continuity of $u^{*}$ implies that for any $t \in D$ and any $\epsilon>0$ there exists an open neighborhood $U_{t, \epsilon} \subset B(0, R)$ of $t$ such that $u^{*} \leq \epsilon+\log C_{V}$ in $U_{t, \epsilon}$. We denote $\cup_{t \in D} U_{t, \epsilon}$ by $U_{\epsilon}$. We have $c^{*}(D) \leq c^{*}\left(U_{\epsilon}\right)=c\left(U_{\epsilon}\right)$. There exists a compact set $K_{\epsilon} \subset U_{\epsilon} \subset B(0, R)$ such that $c\left(K_{\epsilon}\right) \geq c\left(U_{\epsilon}\right) / 2 \geq c^{*}\left(E_{R}\right) / 2$. Hartogs lemma gives to us $n_{\epsilon} \in \mathbb{N}$ such that

$$
\frac{1}{n} \log \left|P_{n}(t)\right| \leq 2 \epsilon+\log C_{V} \quad \forall n \geq n_{\epsilon} \quad \forall t \in K_{\epsilon} .
$$


We can extend the inequality to $\mathbb{C}$ using the Bernstein-Walsh lemma:

$$
\frac{1}{n} \log \left|P_{n}(t)\right| \leq 2 \epsilon+\log C_{V}+\frac{h(n)}{n} V_{K_{\epsilon}}^{*}(t) \quad \forall n \geq n_{\epsilon} \quad \forall t \in \mathbb{C} .
$$

The main difficulty is that this inequality depends on $K_{\epsilon}$ and $K_{\epsilon}$ depends on $\hat{f}$. We will estimate $V_{K_{\epsilon}}^{*}(t)$ in terms of $c\left(K_{\epsilon}\right)$ in order to find a uniform bound. Since $K_{\epsilon} \subset B(0, R)$ we have $V_{|t| \leq R}^{*}(t) \leq V_{K_{\epsilon}}^{*}(t)$. The behavior of the Green's function at $\infty$ is well known:

$$
\left\{\begin{array}{l}
V_{|t| \leq R}^{*}(t)=\log |t|-\log c(|t| \leq R)+o(1) \\
V_{K_{\epsilon}}^{*}(t)=\log |t|-\log c\left(K_{\epsilon}\right)+o(1)
\end{array}\right.
$$

where $o(1)$ represents a function tending to 0 when $t \rightarrow \infty$. We deduce by removing the singularity at $\infty$ that

$$
h(t)=V_{K_{\epsilon}}^{*}(t)-V_{|t| \leq R}^{*}(t)
$$

is harmonic and positive in $|t|>R$. We denote $\tau_{\mathbb{P}^{1}(\mathbb{C}) \backslash(|t| \leq R)}$ by $\tau$. By definition of Harnack distance we have $h(t) / h(\infty) \leq \tau(t, \infty)$. The function $\tau$ is continuous and then it's bounded in compact sets (in $\mathbb{P}^{1}(\mathbb{C}) \backslash(|t| \leq R)$ ). Then there exists $M>0$ such that $h(t) / h(\infty) \leq M$ if $|t| \geq 2 R$. The constant $M$ depends only on $R$ and then on $E$. By developing $h(t)$ we obtain

$$
V_{K_{\epsilon}}^{*}(t) \leq V_{|t| \leq R}^{*}(t)+M \log \left(\frac{c(|t| \leq R)}{c\left(K_{\epsilon}\right)}\right) \quad \text { if }|t| \geq 2 R .
$$

Combining the previous results we obtain for $|t| \geq 2 R$ and $n \geq n_{\epsilon}$

$$
\frac{1}{n} \log \left|P_{n}(t)\right| \leq 2 \epsilon+\log C_{V}+\frac{h(n)}{n}\left(V_{|t| \leq R}^{*}(t)+M \log \left(\frac{2 R}{c^{*}\left(E_{R}\right)}\right)\right) .
$$

By using $V_{|t| \leq R}^{*}(t)=\log ^{+}(|t| / R)\left(\log ^{+}=\max (\log , 0)\right)$ and the maximum modulus theorem we extend the inequality to $n \geq n_{\epsilon}$ and $t \in \mathbb{C}$ :

$$
\frac{1}{n} \log \left|P_{n}(t)\right| \leq F_{V}+\frac{h(n)}{n}\left(\log 2+\log ^{+}(|t| / 2 R)+M \log \left(\frac{2 R}{c^{*}\left(E_{R}\right)}\right)\right) .
$$

The constant $F_{V}$ is equal to $2 \epsilon+\log C_{V}$. We obtain

$$
\left|P_{n}(t)\right|^{\frac{1}{n}} \leq C_{V} \frac{1}{T_{E}}\left(1+|t|^{\frac{h(n)}{n}}\right) \quad\left(n \geq n_{\epsilon}, t \in \mathbb{C}\right)
$$

for some $T_{E}>0$ by simplification. We deduce that $\hat{f}$ converges in

$$
W\left(C_{V}\right) \stackrel{\text { def }}{=}\left(|x|<\frac{T_{E}}{C_{V}\left(1+|t|^{A}\right)}\right) .
$$


We have $G\left(W\left(C_{V}\right)\right)>0$. Since $1 / C_{V} \geq G_{V} / \kappa$ then $\hat{f}$ converges in

$$
W \stackrel{\text { def }}{=}\left(|x|<\frac{T_{E} G_{V}}{\kappa\left(1+|t|^{A}\right)}\right) .
$$

The constant $L_{E}=T_{E} / \kappa$ verifies that $G_{W} \geq L_{E} G_{V}$. If $G_{V}=\infty$ then $W=$ $\mathbb{C}^{2}$.

ProOF OF 6.2. Up to a linear transformation we can suppose that $V$ is contained in $|x|<1 /\left(1+|t|^{A}\right)$, then it's enough to prove the result for $V=$ $\left(|x|<1 /\left(1+|t|^{A}\right)\right)$.

Suppose the result is not true, then there exists an open neighborhood $W$ of $x=0$ such that for $\hat{f} \in \mathbb{C}[t][[x]]_{A, B}$ convergence on $E$ in $V$ implies convergence in $W$. But there exists $C>0$ (proof of Proposition 6.1) such that any series $\hat{f}=\sum_{n \geq 0} P_{n}(t) x^{n}$ converging in $W$ verifies that

$$
\left|P_{n}(t)\right|^{\frac{1}{n}} \leq \frac{1+|t|^{\frac{h(n)}{n}}}{C} \text { for all } n \geq n_{\hat{f}} .
$$

We fix $n \in \mathbb{N}$. We choose a $F_{\sigma}$ set $F_{n} \subset \mathbb{C}$ such that $E \subset F_{n}$ and $0<c\left(F_{n}\right) \leq 1 / n$. The set $F_{n}$ is of the form $\cup_{m \in \mathbb{N}} K_{n, m}$, where $K_{n, m}$ is a compact set for all $(n, m) \in \mathbb{N} \times \mathbb{N}$. We can suppose that $K_{n, m} \subset K_{n, m+1}$ for all $m>0$ and that all the sets $K_{n, m}(m>0)$ are non-polar. For any set $K_{n, m}$ and any $r>0$ we choose a Fekete polynomial [7] (p. 155) $q_{r}^{n, m}$ associated to $K_{n, m}$ and of degree $r$. By Bernstein's lemma (see [7] p. 156) we have

$$
\left(\frac{\left|q_{r}^{n, m}(t)\right|}{\left\|q_{r}^{n, m}\right\|_{K_{n, m}}}\right)^{\frac{1}{r}} \geq e^{V_{K_{n, m}}^{*}(t)}\left(\frac{c\left(K_{n, m}\right)}{\left.\delta_{r}\left(K_{n, m}\right)\right)}\right)^{\tau_{\mathbb{P} 1}(c) \backslash K_{n, m}(t, \infty)}
$$

if $t \notin K_{n, m}$. We have $V_{K_{n, m}}^{*}(t)=\log |t|-\log c\left(K_{n, m}\right)+o(1)$; let $R_{n, m}$ be a positive number such that $K_{n, m} \subset B\left(0, R_{n, m}\right)$ and

$$
V_{K_{n, m}}^{*}(t) \geq \log |t|-\log c\left(K_{n, m}\right)-\log 5
$$

for $|t|>R_{n, m}$. We have

$$
\left(\frac{c\left(K_{n, m}\right)}{\delta_{r}\left(K_{n, m}\right)}\right)^{\tau_{\mathrm{p} 1}(\mathrm{c}) \backslash K_{n, m}}{ }^{(t, \infty)} \rightarrow 1
$$

when $r \rightarrow \infty$, the limit is uniform in $t \in \mathbb{P}^{1}(\mathbb{C}) \backslash B\left(0, R_{n, m}\right)$. In consequence there exists $r_{n, m}$ such that if $r \geq r_{n, m}$ then

$$
\left(\frac{\left|q_{r}^{n, m}\right|}{\left\|q_{r}^{n, m}\right\|_{K_{n, m}}}\right)^{\frac{1}{r}} \geq \frac{|t|}{10 c\left(K_{n, m}\right)} \quad \text { for } t \in \mathbb{C} \backslash B\left(0, R_{n, m}\right) .
$$


We choose a strictly increasing sequence (in $m$ ) $\left\{s_{n, m}\right\}_{m \in \mathbb{N}}$ such that $h\left(s_{n, m}\right) \geq$ $r_{n, m}$ for all $m \geq 0$. The series

$$
g_{n}(x, t)=\sum_{m>0}\left(\frac{q_{h\left(s_{n, m}\right)}^{n, m}}{\left\|q_{h\left(s_{n, m}\right)}^{n, m}\right\|_{K_{n, m}}}\right)(t) x^{s_{n, m}} \in \mathbb{C}[t][[x]]_{A, B}
$$

converges on $F_{n}$ in $|x|<1$ and then in $V \subset(|x|<1)$. By hypothesis $g_{n}(x, t)$ converges in $W$; if $|t| \geq R_{n, m}$ and $m \geq n_{g_{n}}$ we have

$$
\left(\frac{|t|}{10 c\left(K_{n, m}\right)}\right)^{\frac{h\left(s_{n, m}\right)}{s_{n, m}}} \leq \frac{1+|t|^{\frac{h\left(s_{n, m}\right)}{s_{n, m}}}}{C} \Rightarrow C \leq\left(10 c\left(K_{n, m}\right)\right)^{\frac{h\left(s_{n, m}\right)}{s_{n, m}}} .
$$

Moreover $C \leq(10 / n)^{A}(n>0)$ since $\lim _{m \rightarrow \infty} c\left(K_{n, m}\right)=C\left(F_{n}\right) \leq 1 / n$ and $\lim _{m \rightarrow \infty} h\left(s_{n, m}\right) / s_{n, m}=A$. That contradicts $C>0$.

Next we will see that there are pluri-polar sets such that convergence on their lines implies convergence. In particular, the existence of mixed sets will be proved.

Proposition 6.3. For any $n \in \mathbb{N}$, there exists a pluri-polar set $E \subset \mathbb{C}^{n}$ such that any $\hat{f} \in \mathbb{C}\left[x_{1}, \ldots, x_{n}\right][[x]]_{A, B}$ converging on the lines in $E$ is also convergent in a neighborhood of $x=0$.

Any pluri-polar dense $G_{\delta}$ set (a $G_{\delta}$ is a countable intersection of open sets) is valid for our purposes.

Proof. We define $B=(\mathbb{Q} \times \mathbb{Q}) \cap \bar{B}(0,1) \subset \mathbb{C}$; this set is dense in $\bar{B}(0,1)$ and countable. We choose a sequence $\left\{a_{i}\right\}_{i \in \mathbb{N}}$ numbering $B$. We consider the function $u=\sum_{i \in \mathbb{N}} \log \left|z-a_{i}\right| / 2^{i}$. The function $u$ is subharmonic in $\mathbb{C}$ ([7] p. 41). The function $v\left(z_{1}, \ldots, z_{n}\right)=u\left(z_{1}\right)$ is a pluri-subharmonic function, defined over $\mathbb{C}^{n}$, such that $v \not \equiv-\infty$ and $B^{n} \subset(v=-\infty)$. The set $E \stackrel{\text { def }}{=}(v=$ $-\infty)$ is by definition pluri-polar, and it is also a $G_{\delta}$ set, because $v$ is upper semi-continuous and $(v=-\infty)=\cap_{n \in \mathbb{N}}(v<-n)$.

The series $\hat{f}$ converges on a $F_{\sigma}$ set of lines (Remark 3.1). We claim that any $F_{\sigma}$ set containing $E$ has not empty interior. This would prove the result by Theorem 3.1.

Let $F$ be a $F_{\sigma}$ set of $\mathbb{C}^{n}$ containing $E$. The set $\mathbb{C}^{n} \backslash E$ is also a $F_{\sigma}$ set because $E$ is a $G_{\delta}$ set. The sets $F$ and $\mathbb{C}^{n} \backslash E$ can be written as

$$
F=\cup_{j \in \mathbb{N}} F_{j} \quad, \quad \mathbb{C}^{n} \backslash E=\cup_{j \in \mathbb{N}} D_{j} \quad \text { where } \quad \forall j \quad \overline{F_{j}}=F_{j} \text { and } \overline{D_{j}}=D_{j} .
$$

The sets $D_{j}(j \in \mathbb{N})$ are nowhere dense in $\bar{B}(0,1)^{n}$ because $E$ is dense in $\bar{B}(0,1)^{n}$. Since $\mathbb{C}^{n}=E \cup\left(\mathbb{C}^{n} \backslash E\right)=\cup_{i \in \mathbb{N}} F_{i} \cup \cup_{i \in \mathbb{N}} D_{i}$ then Baire's theorem assures the existence of $F_{j_{0}}\left(j_{0} \in \mathbb{N}\right)$ with non-empty interior. Thus the interior of $F$ is non-empty. 


\section{REFERENCES}

[1] J. ECALLE, Théorie itérative: introduction à la théorie des invariants holomorphes, J. Math. Pures Appl. 54 (1975), 183-258.

[2] M. K. KLimeK, "Pluripotential theory", Oxford, 1991.

[3] R. PÉREZ MARCO, Convergence or generic divergence of Birkhoff normal form, preprint, UCLA, 2000. http://xxx.lanl.gov/abs/math.DS/0009028.

[4] R. PÉREZ MARCO, Linearization of holomorphic germs with resonant linear part, preprint, UCLA, 2000. http://xxx.lanl.gov/abs/math.DS/0009030.

[5] R. PÉrez MARCO, A note on holomorphic extensions, preprint, UCLA, 2000. http://xxx.lanl.gov/abs/math.DS/0009031.

[6] R. PÉrez Marco, Total convergence or general divergence in Small Divisors, Comm. Math. Phys. (223) 3 (2001), 451-464.

[7] T. RAnsford, "Potential theory in the complex plane", London Mathematical Society, Student Texts 28, Cambridge University Press, 1995.

[8] A. SEIDENBerg, Reduction of singularities of the differential equation $A d y=B d x$, Amer. J. Math. 90 (1968), 248-269.

[9] M. TsusI, "Potential theory in modern function theory", Maruzen, Tokyo, 1959.

UCLA, Dept. of Mathematics 405 Hilgard Ave., Los Angeles CA90095-1555, USA

javier@math.ucla.edu 